\title{
Performance Analysis Survey of Various SAR Image Despeckling Techniques
}

\author{
A.Rajamani \\ Department of ECE \\ PSG Polytechnic College \\ Coimbatore, India
}

\author{
V.Krishnaveni \\ Department of ECE \\ PSG College of Technology \\ Coimbatore, India
}

\begin{abstract}
Over the past four decades, the Synthetic Aperture Radar (SAR) imagery has become a beneficial and important application over the optical satellite imagery because of its ability to operate in any weather conditions. However, these images are affected with granular noise termed as Speckle noise. This noise affects the overall quality of the image adversely and hence hinders the observation of vital and crucial information present in the image. Thus, it has become essential to remove this speckle noise using suitable techniques. This paper presents the various important techniques available till date for the removal of speckle noise from SAR images and each technique with its own advantages and limitations are described. It also presents qualitative and quantitative measures of various techniques.
\end{abstract}

\section{Keywords}

Synthetic Aperture Radar imagery, Speckle noise, Denoising, Wavelet Transform

\section{INTRODUCTION}

The Synthetic Aperture Radar (SAR) is very useful for providing information about earth's surface by using the relative motion between antenna and its target. It has various applications such as remote sensing, resource monitoring, navigation, positioning and military commanding, highresolution remote sensing for mapping, search and rescue, mine detection, surface surveillance and automatic target recognition [1]. It possesses numerous advantages over optical satellite imagery; such as, effective operation is achieved irrespective of the weather conditions and can able to penetrate clouds, forest canopy and soil [12]. In spite of all these advantages, it is being affected mostly by the signal dependent noise known as speckle noise, which in turn affects radiometric resolution [28]

In this paper various despeckling techniques right from spatial, frequency domain and up to recent advancements have been addressed in a detailed manner with its own merits and demerits. As there is lot of scope in effectively denoising speckle, researchers still have wide opening to find out a challenging algorithm to meet up all the constraints

Section 2 discusses about the noise model and its modes of representation. The details about the techniques and the comparison of the earlier models to the latest ones in view of speckle suppression in SAR imagery is explained in Section 3. Section 4 gives the results and discussion of various filters and Section 5 gives the conclusion.

\section{NOISE MODEL}

The noise model should be studied for all kinds of noise before preprocessing, for easy application of noise removal algorithm. Speckle is exactly not a noise but is more of granular pattern. The coherent integration of returned scattered energy and that of returned randomly distributed scattered energy causes interference and ultimately introduces speckle noise and it will record both amplitude and phase values of back scattered radiation[47]

In general there are two basic modes of noise as additive and multiplicative. The additive noise is systematic, easily modeled [45] and can be removed easily with lesser efforts. Whereas multiplicative noise which is caused by de-phased echoes from scatters is image dependent, complex to model and also difficult to reduce although it contains useful information [27]

The multiplicative speckle noise is in the form of

$$
\mathrm{Y}(\mathrm{k}, \mathrm{l})=\mathrm{S}(\mathrm{k}, \mathrm{l}) \mathrm{N}(\mathrm{k}, \mathrm{l})
$$

Where $Y(k, l)$ represent the intensity format of corrupted SAR image, $S(K, l)$ represents the noise free SAR image that has to be recovered and $N(k, l)$ denotes the multiplicative speckle component. The logarithmic representation of the multiplicative noise is in turn converted into additive noise which is given by

$\mathrm{Y}(\mathrm{k}, \mathrm{l})=\mathrm{S}(\mathrm{k}, \mathrm{l})+\mathrm{N}(\mathrm{k}, \mathrm{l})$

The log transformation yields undesired effects in the SAR im ages since the mean of the log transformed speckle is not zero. Speckle noise removal is a preprocessing task which involves segmentation, detection and classification of SAR image and the presence of speckle affects radiometric resolution and makes it difficult for human interpretation. So it is mandatory to remove speckle from SAR images for the image processing community, for performing better post processing operations on SAR images and for effective human interpretation too.

\section{TECHNIQUES FOR SPECKLE NOISE REMOVAL OF SAR IMAGES}

The three main aims of Speckle filtering are noise removal in uniform regions, preserving and enhancing edges without changing features, and to provide a good visual appearance. The speckle filtering involves three main stages which is mainly adopted for transform based speckle reduction approach that transforms the original space or domain into new domain, manipulation of co-efficient and transform the resultant co-efficient back In to the original domain.

Multi look processing is the technique which averages several independent images or the number of looks (L). The SAR image is processed using digital image processing either in frequency domain mostly by wavelet transform or in the spatial domain by manipulating the neighborhood pixels.Multi look integration technique has a drawback, there is an increase in computation when number of looks (L) is 
increased and also degradation of image resolution happens with smoothing of uniform areas. The two SAR Speckle reduction techniques that were widely used are multi look processing and adaptive image restoration techniques [27].

Adaptive image restoration on the other hand uses post image formation technique which makes use of filters like Lee filter, Local statistics filters, point wise linear filters and Maximum A Posteriori (MAP) filter.

\subsection{Spatial domain filtering techniques}

Spatial domain filtering involves the direct manipulation of pixels by using kernel mask or window runs over the entire image. The advantages of this method are that it is very good for contrast enhancement and image sharpening. The disadvantage of this technique is that it shifts boundaries during sharpening and only manipulates pixel and also no frequency information is achieved.

Spatial domain filters such as Lee, Frost, Improved LeeSigma, Mean, Median, Gamma-MAP, Wiener, Kuan, probablisitic patch based and Kalman filters have their own common limitation of fixed local window size and orientation. Also usage of neighborhood pixel values weakens the object signal and edge information. Even though statistical filters are good in speckle reduction, they lose important feature details and moreover prior knowledge about noise statistics is required. The Lee and Kuan filters are multiplicative models formed by a linear combination of local mean and observed pixel. Lee and Kuan filters are evaluated based on performance metric of Minimum Mean Square Error criterion (MMSE) and is optimal when detected intensities are Gaussian distributed. The MAP filter can be better implemented for speckle removal in forested areas and agriculture land with Gamma Probability Density Function model. The Lee, probablisitic patch based and MAP filters could also have the limitations that include noise removal only for uniform area without edges and introduction of blurring which in turn results in degradation of resolution if the window size increases beyond $3 \times 3$ [2.] The qualitative and quantitative analyses for various spatial filters are described in detail considering many performance metrics and are presented below.

The pixel value estimate of Lee filter is calculated as $\hat{x}=\frac{\bar{y}+\sigma x^{2}(y-\bar{y})}{\left(\bar{y}^{2}+\sigma^{2} x\right) / L}$ and same for Kuan filter is given by

$$
\hat{x}=\bar{y}+\frac{\sigma x^{2}(y-\hat{y})}{\sigma x^{2}+\left(\bar{y}^{2}+\sigma^{2} x\right) / L} \text { and } \sigma x^{2}=\frac{L \sigma y^{2}-\bar{y}^{2}}{L+1} \text {. }
$$

If $L=1$, Nathan filter[16] is obtained which is a special case of Kuan filter and has the limitation of its applicability only to one look SAR images and its estimate of pixel value is known from the formula $\hat{x}=\frac{\bar{y}+\sigma x^{2}(y-\hat{y})}{\sigma x^{2}+\left(\bar{y}^{2}+\sigma^{2} x\right)}$ where $\sigma x^{2}=\frac{\sigma y^{2}-\bar{y}^{2}}{2}$.The exponential impulse response of Frost Filter is known by the formula $m=\exp \left(-k c_{y}\left(t_{o}\right) / t\right)$ where $c y=\frac{\sigma y}{\bar{y}}$.The pixel value at location $(\mathrm{m}, \mathrm{n})$ for Kalman filter is estimated as $\mathrm{X}(\mathrm{m}, \mathrm{n})=\varepsilon_{(p, q) s w} a_{p q} x(m-p, n-q)+u(m, n)$ where $u(m, n)$ represents noise sequence[16]The Iterative Conditional Modes (ICM) filter is the extension of point wise MAP filter to develop block filtering with grouping of observations with similar grey levels. Frost filter exhibits worst visual quality due to the removal of only few numbers of speckles. But it is better in terms of SNR and PSNR due to less number of noises, better Edge Preserving Ability (EPA), since it leaves more noise unremoved, including information mistakenly detected as edge. The Frost filter is not optimal but is used for some applications [36].

Mean filter is a low pass filter which not only smoothens but also smears the edges and fine details. Moreover a mean and median filter is not adaptive filters and also does not perform well for high density noise. So Adaptive noise smoothing Filter has attracted researchers and was proposed by the Authors [10].It is a point processor which adapts to local changes. It outperformed earlier method by using Fast Fourier Transform (FFT) which also yields blurring effect. In this paper Non Stationary Mean and Non stationary Variance (NMNV) image model with a Local Linear Minimum Mean Square Error (LLMMSE), to overcome the above problem has been implemented[43].The limitation of Lee - Sigma filter is due to fixed sigma, which causes blurring of low contrast edges and linear features. Later, improved Lee sigma filter has been used to overcome the blurring and shows improved performance by decreasing computational complexity. The comparison of several filters uch as Lee, Kuan, Enhanced Lee, Frost, Enhanced Frost[28] and Gamma MAP were carried out and results as all filters show some tradeoff between speckle reduction and presentation of useful information[14].

The Heuristic filter like moving average filter, geometrical or Morphological filters too have limitations of blurring, fixed window size and applicability only for homogenous areas.

It is [45] proposed that reduction of speckle based on number of independent amplitude values have certain limits. This method has a limitation as Signal to Noise Ratio (SNR) improvement is good only for low resolution (small aperture) transducers but lower for high resolution (large aperture) transducers. Also Computational complexity will be increased during individual pixel by pixel processing.

Then, the method using computer simulation of image pixels had been carried out. Normally the phase and amplitude of the pixel varies and it can be reduced by averaging the phase and amplitude of the pixel variations [45] or by performing median filtering of azimuth looks per pixel. This method has also got a limitation as averaging results in over smoothing and in turn results in blurring effect.

A new technique of homomorphic approach (Bhuiyan, 2007) was suggested wherein the SAR image is log transformed to make multiplicative noise into additive which again results in blurring of many important features.

A solution for the above problem was introduced by using adaptive noise smoothing filter for Image with signal dependent noise. It is a point processor which adapts to local changes. [2] It overcomes the above said problem of blurring by using two dimensional recursive filtering and it also possesses the advantage of less computation time and handles space-variant blur easily. Non stationary mean - non stationary variance image model with Local Linear Minimum Mean Error (LLMMSE) filter has been used in this approach[10]. The modified approach had been suggested for the above as [45] the use of adaptive filters with two thresholds, one for the function of image parameters and another as fixed. The modified adaptive filter 
averages homogeneous areas and also preserves the edge and textual information.

One more method had been proposed for speckle reduction by correlating two measurements made at different spatial positions [45] and by computing the optimum aperture displacement. However, by using this method it is difficult to retrieve small details.

The alternate solution for speckle reduction [45] which makes use of the anisotropic diffusion which is commonly employed to choose conduction coefficients for smoothing edges and boundaries are also found.

The authors have suggested that it might be possible to process the whole image in a single step [45] or by partitioning using Zero Adjustment Procedure (ZAP).

Other standard filters like kalman, geometric, Oddy and Adaptive Filter on Surfaces (AFS) filters have limitation of algorithmic complexity. Kuan filter reconstruction estimate is given by.

$$
\hat{x}=\frac{(\alpha-L-1) \bar{y}+\sqrt{\hat{y}^{2}(\alpha-L-1)^{2}}+4 \alpha L y \bar{y}}{2 \alpha}
$$

Where

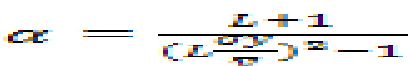

The oddy filter is a mean filter and its shape varies based on local statistics.

$$
\begin{aligned}
& \hat{x}=\bar{y} \text { if } m<\alpha \bar{y} ; \hat{x}=\frac{s_{k} s_{l} w_{k l} \cdot y(k, l)}{s_{k} \varepsilon_{l} w_{k l}} \text { if } m>\alpha \bar{y} \\
& w_{k l}=1 \text { if }|y(k, l)-y| \leq m \quad w_{k l}=0
\end{aligned}
$$

Otherwise $m=1 / 8 \varepsilon_{\mathrm{g}} \varepsilon_{\mathrm{g}}\|y(k, I)-y\|$ where $\alpha \rightarrow$ filter parameter and $\mathrm{w} \rightarrow$ adaptive binary mask

The reconstructed estimate is given by

$$
\mathrm{x}=\frac{\sum_{\mathrm{k}} \sum_{1} w_{\mathrm{ki}} y_{\mathrm{k}} \mathrm{j}}{\Sigma_{\mathrm{k}} \Sigma_{1} w_{\mathrm{kl}}}
$$

The above limitation can be overcome by adaptive filter which suggests homomorphic technique (i.e.) multiplicative noise $\log$ transformed into additive noise and estimate the original image by performing exponential operation which in turn results in poor reconstruction by losing important information.

Later people started using Frequency domain techniques using the wavelet \& curvelet transforms based speckle reduction since those techniques provide more information and has been suggested by many researchers and has been described below.

\subsection{Frequency domain filtering techniques: (Wavelet, Curvelet and Directionlet \\ Transform)}

This domain uses manipulation of frequency and it is found to be good for periodic noise reduction and for image sharpening. The main drawback is that it is not suitable for contrast enhancement and manipulates frequency only. The following frequency domain filters exhibit their own merits and demerits.
In order to obtain frequency, phase and amplitude information, it has been preferred to use Complex wavelet transform. Complex - valued extension to the real wavelets and to the standard discrete wavelet transform is known as complex wavelet transform. There are two types; redundant and non-redundant complex wavelettransform. SAR image despeckling using complex wavelet transform is advantageous over discrete wavelettransform as they are applicable for Multi-Resolution Analysis (MRA) and useful for sparse representation [2], characterization of the structure of an image and it also possesses high degree of shift invariance in its magnitude, but it has a drawback, that it exhibits $2 \mathrm{~d}$ ( $\mathrm{d}=$ dimension of the signal being transformed) redundancy when compared to Discrete Wavelet Transform (DWT).

Non redundant DWT for non stationary signal processing application has also got many drawbacks such as, no translation invariance i.e. loss of many important coefficients during translation from original signal to sub bands[46] Whether discrete or undecimated wavelet, the biggest problem is the selection of optimal thresholding, small noise distribution, mismatch at different scales, shift sensitivity, poor directionality and absence of phase information.Here the history behind the development in frequency domain techniques right from Fourier transform to curvelet[30]complex wavelets techniques including recent developments have been presented.

Earlier, Fourier transform mathematical approach was used, but the drawback in using Fourier transform is that it can give only time localization or frequency localization at one time. It is not possible to get both the information simultaneously. Later on, Short time Fourier transform came into practice and has the limitation of fixed window size, inability to change window size, Hence it is not meant for Multi Resolution Analysis. To overcome all these problems wavelet transform has been used since it is a powerful tool which supports MRA[2]. In wavelet sub bands, noise is present in small co-efficient and feature details are present in large coefficients. But it also has got the drawback of shift variance and poor directional selectivity. It has three main steps:

1. Calculate wavelet transform of noisy image

2. Manipulate the wavelet coefficients

3. Compute inverse transform [35]

Shape of the wavelet is chosen based on the different features to extract. The wavelet transform allows the representation of a signal onto the orthogonal basis. Each term of the basis represents the signal at a given scale. It is decomposed into basis which in turn gives details and approximation, and can be stored as wavelet coefficient. This representation is called as a wavelet representation. Among the infinite wavelet basis choose the basis which is very close to the feature to be extracted. By using the proportional relation between noise coefficient and wavelet coefficient one can extract linear features such as edges and thin stripes [33].

Since Noise contribution is more in high reflections, normalization is performed which results in equal noise contribution for all targets which does not produce smooth edges and linear features. Asymmetric wavelet selection works well for delineating edges and that of symmetric wavelet selection to enhance thin features. Wavelet shrinking technique has also been used but the drawback is setting zero for the wavelet coefficient which are lower than the 
threshold, which in turn results in the creation of artifacts and modifies the values of analyzed pixel [33].

Wavelet Packet Transform (WPT) and Stationary Wavelet Transform (SWT) are an alternative to DWT, but they also have got the limitation that they are redundant and computationally intensive.

Earlier, Wavelet shrinkage algorithms were used to have dependencies between wave co efficient and the wavelet coefficient of complex back scattered signal of SAR image followed Generalized Gaussian Probability Density Function ((GGPDF), Later Cauchy PDF has been used because of unimodel and symmetric. The Cauchy PDF is used for modeling the co-efficient of log transformed reflectance[26]Wavelet Embedded Anisotropic Diffusion (WEAD) avoids single space representation of SAR image either in the time domain or in the frequency domain since that representation is not sufficient for better interpretation of the image. So, multi scale wavelet transform was developed. WEAD is a Bi stage filter where first stage involves partial differential equation based anisotropic diffusion and the second stage employs wavelet based bayesian shrinkage. The wavelet thresholding technique is better than adaptive restoration method. The Spatially Adaptive Wavelet Based Bayesian Minimum Mean Absolute Error Estimator or Bayesian MAP (SA-WBMAP) and wavelet based Hidden Markov Tree (HMT) model are used. In this method/experiment [26], the dispersion parameter of bivariate Cauchy PDF is estimated using Gauss hermite quatrature technique but it has the limitation of high computational complexity (15 times more).

Instead of the above method, Bivariate Cauchy PDF with MAP [26] estimation provides higher FOM (Figure of Merit) values, better edge preservation, increase in ENL (Equivalent Number of Looks) and better performance in terms of PSNR.

Depending on the nature of the wavelet coefficient, the thresholding types have been classified as universal, bayesion shrink and wavelet threshold. All thresholding techniques are finally compared with spatial domain filters and have been proved better than spatial filters. Drawback in the wavelet threshold is that still fails in providing a better result in preserving edge information [24].

In order to overcome the above limitation a new approach, Insitu Single - Pointed Wavelet based (ISPW) method had been used for distributing approximate part of wavelet coefficient that operate on both approximate and detailed components and also[24] loss of edge is avoided. This method has got many advantages, for example it does not affect features of other useful signals, preserves the edges to the maximum extent and it also avoids the problem of over filtering that in turn causes loss of information. Edge Preserving Ability (EPA), visual quality, PSNR, SNR shows better result, removes more speckle and also possesses less radiometric resolution. A possible limitation might be less Equivalent No.of Looks (ENL) but this can be further improved upon.

$$
\left(\frac{\text { mean }}{\text { standard deviation }}\right)^{2}=L=\text { constant }
$$

(Measure of SNR)

(5)

Another wavelet approach was introduced [19] using hard and soft thresholding of wavelet coefficients of $\log$ transformed image with Automatic Target Detection Recognition (ATDR) problem. Soft thresholding gives better result than hard thresholding because it is computationally efficient. This method used Polari metric Whitening Filter
(PWF) along with wavelet shrinkage method. The limitation of this approach is that it does not utilize spatial correlation and only the correlation across polarizations is used. Moreover, if the wavelet filter length is more, the detail of the image is over smoothened and which in turn increases computational complexity.

The use of orthogonal wavelet domain for speckle reduction and enhancement of SAR images and also three methods were suggested [29] as soft thresholding of wavelet coefficients, enhanced adaptive Lee filter and adaptive sigmoid thresholding. Sigmoid function is similar to activation function used in neural networks. In a $256 * 256$ gray scale noisy image given to orthogonal DWT, the parameters are chosen and finally the inverse DWT is taken to get the reconstructed image. The limitation of this approach lies in the employment of additional precaution while choosing the type of wavelet, length of wavelet filters and number of decomposition levels. When the number of decomposition level increases, computational complexity increases.

Another approach suggests [29] the use of tree structured filter banks for efficient speckle removal and detection of SAR images. The thresholding methods are namely, soft thresholding of wavelet coefficients, enhanced adaptive Lee filter and adaptive sigmoid thresholding. Four stages of Infinite Impulst Response Quadrature Mirror Filter (IIR QMF) bank decomposition for soft and hard thresholding and five stages for sigmoid method were used and this method helps in preserving resolution with significant improvement in speckle reduction.

To avoid the above said problem, ad-hoc threshold was proposed, wherein a method that uses non ad-hoc with less computational complexity and incorporating spatial dependence is used. This technique employs Bayesian Minimum Mean Absolute Error Estimator (MMAE) or Bayesian MAP estimator[8]Even though it possesses various advantages like improvement in Peak Signal to Noise Ratio (PSNR), Structural Similarity Index Matrix (SSIM), ENL, edge preservation and line structures, it introduces artifacts. One more Bayesian approach was developed to reconstruct signal using a set of compressed sensing measurement affected by heavy tailed noise using multivariate Cauchy prior and shows better result than the Gaussian method.

A new class of Adaptive shrinkage factor for despeckling SAR images was suggested [3]. The above method is easily computable but does not preserve fine details in complex images. This spatially adaptive shrinkage factor has two variable functions: one is the likelihood of the magnitude of noise wavelet coefficients and useful edges and another is the binary mask that indicates the estimated edge positions. Adaptive shrinkage factor can be easily computed using likelihood model.An analysis of the usage of complex wavelets for shift invariant analysis and filtering of signals was done. Complex wavelet coefficients was obtained [37]by using dual tree wavelet filter which is advantageous than traditional wavelet transform in the following ways: Dual Tree Complex Wavelet Transform (DTCWT) provided limited redundancy, shift invariance, good directional selectivity, computationally efficient, filtering multidimensional signals, perfect reconstruction, multiscale decomposition and good frequency response when compared to Discrete Wavelet Transform (DWT).The DWT has many drawbacks such as translation variant, loss of information coefficients during translation from original signal to sub band, need of more storage space and more time required to 
solve and save the coefficients. The biggest limitation of Discrete or Undecimated Discrete Wavelet Transform (UDWT) is the selection of optimal thresholding and then increased computational requirements, high redundancy and expensive even though it provides shift invariance [37] DWT has one more drawback of lack of symmetry of mother wavelet. The shift sensitivity of the DWT is generated by the down samplers used for its computation but UDWT without down samplers provides .high redundancy. Aleksandra Pizwrica and wilfried Philips [3]suggested a new class of adaptive shrinkage estimator for despeckling SAR images. The earlier methods are easily computable but do not preserve fine details in complex images. This spatially adaptive shrinkage factor has two variable functions: one is likelihood of magnitude of wavelet coefficients of noise and useful edge and another is the binary mask that indicates the estimated edge positions. Shrinkage factor can be easily computed using likelihood model.

Another approach for shift invariance and directional selectivity which is designed in frequency domain are laplacian pyramids and steerable filters and separable polyphase filter bank that have better directional selectivity with no redundancy but has the limitation of high shift dependency and unclearity about multiscale decompositions[37]

It has been proposed to remove speckle using wavelet Bayesian denoising combined with Markov Random Field (MRF) modeling [22], which uses a two state Gaussian mixture model for modeling wavelet coefficients and statistical dependence between wavelet coefficients can be known by using Markov random field [8] and noise free coefficients by shrinkage function based on weighted averaging of the Bayesian estimator. This method outperforms the existing standard spatial filters in terms of PSNR and ENL using cycle spinning technique up to $4 \times 4$ shifts. Even though it is better than the best among spatial filters known as refined Lee filter, it has its own limitation as it exhibits high computational complexity and less performance if $8 \times 8$ cycles spinning is used.

Zhang et al. developed wavelet coefficients of natural images and auto correlation function model for signal and noise in the Undecimated Discrete Wavelet Transform (UDWT). It has the advantage of translation-invariance when compared with DWT. The limitation of UDWT is that of orientation variant. The steerable pyramid provides shift invariance which includes translation and orientation invariance[46].

The spatial domain track has also grown up along with wavelet domain by using Local Adaptive Median filter for speckle reduction. The microwave radiation fall on the target and the back scattered return energy causes pixel to pixel variation in intensity which in turn produces salt and pepper granular pattern known as speckle. It is advantageous than all other spatial domain filters by the best way of preserving important edges, linear features and fine details. It uses local statistics instead of global statistics and replacing salt and pepper noise with a local median value and not by derived values. Moreover it replaces one of the neighborhood valid pixel values without changing other pixel values. It outperforms compared to all spatial filters by means of following performance indicators [14].

Speckle Suppression Index (SSI) is the performance measure which gives coefficient of variance and is given by.

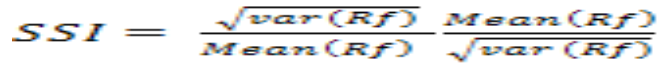

(6)

Where $\mathrm{Rf}$ is the Filtered image value and $\mathrm{R}$ is the original image value. This method gives smaller value compared to other filters and proves better speckle suppression.

Speckle Image Statistical Analysis (SISA), when its mean close to unity shows better result and resulting speckle image (SP) is given by Fang Qiu et al[14].

(7)

Edge Enhancing Index (EEI) is mainly used for knowing edge preservation like boundary between water bodies and land and this measure is given by.

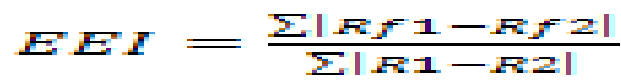

(8)

Where R1, R2 are original pixel values and Rf1, Rf2 are filtered values. This method shows higher EEI than the other spatial filters and thus proves better edge preservation.Feature Preserving Index (FPI) gives the information about Linear features and structures which are better preserved in this method by giving higher FPI compared to other spatial domain filters and this measure can be evaluated by using the formula.

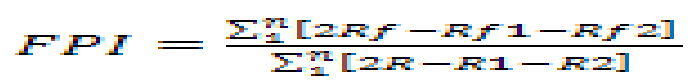

(9)

Image Detail Preserving Coefficients (IDPC) provides correlation between original and filtered image. Higher IDPC indicates better detail preservation. Visual comparison also shows better result and the limitation could be the slightly lower SPI compared to Local sigma filter. Further performance can be improved by increasing the size of the window and the number of iterations[14].

The SAR image generation model generally follows Rayleigh distribution [12]but urban areas of image exhibits heavy tailed distribution (Non Rayleigh).Some alternate distributions like Weibull, log-normal and $\mathrm{K}$ distributions were suggested to meet varying degrees depending on the application. Recently alpha stable distribution has been developed to show the real and imaginary parts of complex wave[14]

Limitation of the above traditional Rayleigh distribution with MMSE filter is that it is not meant for heavy tailed statistics [47].To overcome the above problem, MAP filter based on heavy tailed Rayleigh modeling could be used.

An alpha-stable model for wavelet coefficients was developed [46]by ignoring local correlation structure between coefficients and relation between different scales and orientations like the thresholding methods has been used.

As the thresholding method of denoising is not effective due to the independence between wavelet coefficients with ignorance of important dependency, one more approach was developed based on modeling auto correlation function of wavelet coefficients of logarithmically transformed SAR images incorporating directionality [46] into a steerable pyramid wiener filter. Limitation of this approach being the size and shape of window local indices of neighbor's performance could be improved. 
An extension to the wavelet transform in many directions is proposed as the Directionlet which represents an image sparsely while capturing anisotropic structure. Using directionlet transform [7] and cartoon texture model, the image can be partitioned into independent closets and performed in different directions and provides better performance in terms of visual quality and detail preservation has been observed.

Another image denoising method was suggested employing a model using Bivariate Cauchy probability density function distribution [21] with its local variance in complex wavelet domain. Bivariate MAP estimator is used to estimate $\mathrm{w}$ from the noisy observation and is defined as

$$
\stackrel{w}{w}=\arg _{\mathrm{w}} \max [\operatorname{pn}(\mathrm{y}-\mathrm{w}) \mathrm{pw}(\mathrm{w})]
$$

But it has the limitation that prior knowledge about distribution of clean wavelet coefficients is required along with the PDF of the noise. If the speckle intensity is Gaussian distributed, then the Linear Minimum Mean Square Error (LMMSE) filter is better and for asymmetric distribution, MAP filter is better. Future scope suggested as mixture of MAP and MMSE estimators can be used to improve the performance[21]

Another wavelet based despeckling approach which used the combination of undecimated wavelet transform, an adaptive filter like wiener filter and mean filter [43]. The existing thresholding techniques are sure shrinkage, oracle shrinkage, normal and universal thresholding, visu thresholding, hard and soft thresholding. But a new one named as brute force thresholding has been used. The limitation of UDWT is when saving coefficients, it needs more storage space and needs more time for computation. The brute force thresholding also has the limitation as more time is needed for computation.

In order to solve the above thresholding problem, M.I.H Bhuigan et al have proposed spatially adaptive wavelet based bayesian method using the Cauchy prior zero location model and zero mean Gaussian distribution. Wavelet shrinkage based on Bayesian formalism is more effective than thresholding techniques using prior PDF. In Bayesian estimators the wavelet coefficients are assumed mutually independent but in practice they show dependence[8]

As it is well known that real wavelets have four limitations as shift invariance, poor directional selectivity, aliasing and oscillation, and due to those complex wavelets have come in to practice. The complex wavelet method [11] has been a novel wavelet based denoising method of SAR image using interscale dependency. In this paper, bi variate shrinkage function and MAP filter for enhancing wavelet coefficients are used. Wavelet coefficients are enhanced based on the significance of coefficients by assuming Gaussian distribution. The basic functions for the real and imaginary part of the Dual Tree Complex Wavelet Transform (DTCWT) coefficients are orthogonal which means that they are uncorrelated. New shrinkage function, which depends on both the coefficient and its parent, shows improved results. In this paper it has been considered that the locations of edge or radiometric scattered points modify radiometric scatter points and in turn modify wavelet coefficients. It provides more details in the line and edge area.

One more approach using complex wavelet involves the implementation of the Hyper Analytic Wavelet Transform (HWT) for image denoising. This approach [25] involves application of Hilbert transform to the data and the real wavelet is applied to the analytical signal associated to the input data and ultimately complex coefficients are obtained. Orthogonal or Biorthogonal real mother wavelets are used for the computation of Hyper analytic Wavelet Transform (HWT) and MAP filter is used for filtering. This approach can be enhanced as diversity enhancement and cooperation between parametric and nonparametric and can improve speed optimization.

A 2D GARCH model [35] for speckle suppression in SAR images has been implemented. It is a Bayesian based Daubechies 4 Wavelet (Db4), two level decomposition approaches. The main concept in this approach was arrived at with the knowledge of important characteristics of wavelet coefficients like heavy tailed marginal distribution a dependency between the wavelet coefficients. Thus, the speckle reduction has been performed by using MAP estimator. 1D GARCH was used for modeling financial time series but 2D GARCH modeling deals with intrascale dependences of wavelet coefficients, which is flexible and provides a closed form solution. The histogram of wavelet coefficients is highly accurate using 2D GARCH than the other PDF like Generalized Gausian (GG). For assessing the accuracy of 2D GARCH coefficients, boot strap technique was used.

One more approach [32]was analyzed using sparsity promoting speckle denoising, also called basis pursuit denoising. This paper reviewed wavelet denoising approach and stated that earlier linear approximation of the multiplicative model has been performed without taking logarithm. The general denoising algorithm cannot work on multiplicative noise. It is a usual practice to convert the multiplicative noise into additive noise by logarithm transform. Later on, the linearized image obtained by taking logarithm of the noisy image is taken and applied for Bayesian estimation. Then iteratively attenuated thresholding was performed on the detailed wavelet coefficients. After sometime, instead of linearization, contourlet transform [17]was used to estimate the variance of the noisy image. Currently wavelet methods like Discrete Cosine Transform (DCT), Curvelet transform, contourlet transform, wavelet shrinkage have been used. It has been concluded that among spatial domain denoising methods, Frost filter and that among transform domain like wavelet domain, iterative thresholding gave good results in terms of PSNR.

The idea of implementing Dual Tree Complex Wavelet Transform (DTCWT) based SAR despeckling involving inter scale dependencies between wavelet coefficients [26]was suggested. The DTCWT has been organized as one tree which provides real co-efficient and another one provides imaginary coefficients and so it provides both magnitude and phase information. The coefficients of DTCWT after log transformation at level $\mathrm{j}$ and orientation $\mathrm{i}$ is given by $d_{j}^{i}\left(k_{j} l\right)=x_{j}^{i}\left(k_{j} l\right)+n_{j}^{i}\left(k_{j} l\right)$

This method makes use of Mellin transform to find dispersion parameter of Bivariate Cauchy PDF and using the filter, bivariate MAP which is very much advantageous than earlier methods of numerical integration, is employed/obtained. It has the limitation of increase in decomposition level and causes blurring of despeckled image[26]. 
The modification over the above method has been used with Multivariate PDF with MAP estimation [27] considering both interscale and intrascale dependencies of wavelet coefficients, which provides still better performance in terms of ENL, Peak Signal to Noise Ratio (PSNR) and Mean Structural Similarity index Matrix (MSSIM) metrics.

Even though redundancy provides extra information for analysis, it is at the expense of computational power. Earlier, so many wavelet shrinkage algorithms were used to have dependence between wavelet co-efficient across different scales. It has its own advantages of improved directional selectivity, approximate shift invariance and perfect reconstruction using MAP estimator over DWT. The measure of quality of reconstruction is known by the important metrics which are calculated as below [26]. $\mathrm{ENL}=\mathrm{Mean}^{2} /$ Variance(12)

$$
\operatorname{MSSTM}(X, Y)=\frac{1}{M} \sum_{j=1}^{M} \operatorname{ssIM}\left(x_{j}, y_{j}\right)
$$

Where $\mathrm{X}$ and $\mathrm{Y}$ are the reference and despeckled images respectively. $\mathrm{M}$ is the number of local windows in the images.

$$
\operatorname{ssTM}(x, y)=\frac{\left(2 \mu_{x x} p_{y}+c_{1}\right)\left(2 \sigma_{x y}+c_{z}\right)}{\left(\mu_{x}^{2}+\mu_{y}^{2}+c_{1}\right)}
$$

$\mu_{x}$ and $\mu_{y}$ are the estimated mean intensities, $\sigma_{x}$ and $\sigma_{y}$ are the standard deviation.The constants $c_{1}, c_{2}$ are given as $c_{1}=\left(k_{1} D\right)^{2}$ and $c_{2}=\left(k_{2} D\right)^{2}$, respectively, where $k_{1_{2}} k_{1} \ll 1$ and $\mathrm{D}$ is dynamic range of the pixel values $\sigma_{x y}$ can be estimated as

$$
\begin{aligned}
& \sigma_{x y}=\frac{1}{N-1} \sum_{i=1}^{N}\left(x_{i}-\mu_{x x}\right)\left(y_{i}-\mu_{y}\right)(15) \\
& \text { PSNR }=10 \log _{10} 25^{2} / M M S E
\end{aligned}
$$

It has been proposed that [27] the generalized SAR despecking based on DTCWT, exploits Interscale and Intrascale dependencies. This approach used multivariate Cauchy pdf as model for DTCWT coefficients and is given by

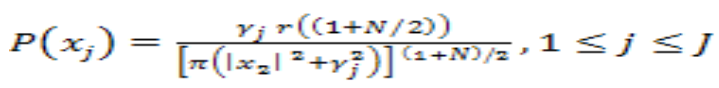

Where $\gamma_{j}$ is the dispersion parameter at jth level

Figure of Merit (FOM) can be used to measure edge preservation and is defined as

$$
P=\frac{100}{\max \left[N_{D E_{a}} N_{I D}\right]} \sum_{i=-1}^{N_{D E}} \frac{1}{1+\beta d_{i}^{2}}
$$

(18)

NID and NDE are ideal edge pixels and number of detected pixels respectively. di is the distance between the ith detected edge pixel and the closest true edge pixel. ${ }^{\beta}$ is the calibration constant.

The dependency between wavelet coefficients, their neighbors and coefficients across scales have been considered. The heavy tailed behavior of the signal is modeled by multivariate Cauchy PDF. The Mellin transform has been used to find the dispersion parameter. Six directional selective sub bands are used. The intrascale and interscale dependencies of the DWT coefficient values can either represent median or any coefficient in a window size. Finally inverse DTCWT has been used to get despeckled image. The biased mean is connected by subtracting the mean of the log transformed speckle from the image obtained after inverse DTCWT and then exponential transformation is performed. This paper presents three levels of decomposition and further decomposition will result in over blurring and moreover more dependencies will add interference to the algorithm which will increase the computational complexity[27].

Krishna Mohan et al. used curvelets over DTCWT and traditional filters for improved denoising of SAR speckle image and have high PSNR when compared to other techniques.

It [41]has been proposed using Fusion techniques for despeckling oil spill SAR image. All statistical filters perform better speckle reduction but with loss in details whereas hybrid filters can preserve details well but the effect of speckle reduction is not good. To make use of the advantages of both filters a Fusion method has been implemented by combining Frost and Relaxed Median filter to extract better performance.

Approach of Fusion technique achieved by Guozhong chen by combining Wavelet transform and statistical filter by having the advantages of good detail preservation and best speckle reduction respectively. An improved wavelet based denoising using data fusion technique gives better results compared to other single filter.

One more Fusion approach [23] has been given by merging wavelet bayesion technique with Markov-random-field-based image regularization has been given. In this paper wavelet coefficients are modeled by two-state Gaussian mixture model and spatial dependence by Markov random field. This fusion technique outperformed standard wavelet denoising techniques in terms of SNR and ENL and shows better performance than Refined Lee filter.

Non Local Means (NL-means) is a new measure for denoising and is based on non local averaging of all pixels in the image. Local averaging filters like Gaussian smooth filtering, anisotropic filtering, total variation minimization approach and neighborhood filtering exhibit their own limitations. Gaussian has the limitation that it is optimal in flat parts of the image but edges and texture are blurred. The anisotropic filter and total variation minimization approach are preserving edges while flat and textured regions are degraded. The drawback of neighborhood filtering is that it compares only gray level values in a single pixel, which is not advisable. The alternative to this local approach is NL means which is not only compares grey level in a single point but also whole neighborhood and performs well than local method in terms of method noise, visual quality and MSE [39].Recently people have become interested in solving the denoising problem using advanced image processing techniques like statistical modeling of multiplicative noise with Mellin framework considering only positive random variables and their multiplication. One more approach in spatial domain filtering is also being used recently as patch based non-local method since images are highly redundant in nature. Compressed sensing and Graph cut method is also coming up for effective noise removal by designing efficient, minimum cut algorithms with total variation minimization model [15]. 


\section{RESULTS AND DISCUSSION}

Here is the performance comparison analysis of spatial and wavelet domain filters for the SAR image of river with respect to various metrics like RR,ENL,SNR,PSNR,EPA given qualitatively and quantitatively as well. Fig.1 shows qualitative results of various filters. Table 1 compares the metric wise performance analysis of various filters, Table 2 summarizes various methods of denoising, Table 3 lists the limitations of various denoising approaches and Table 4 enlists the advantages of various denoising approaches

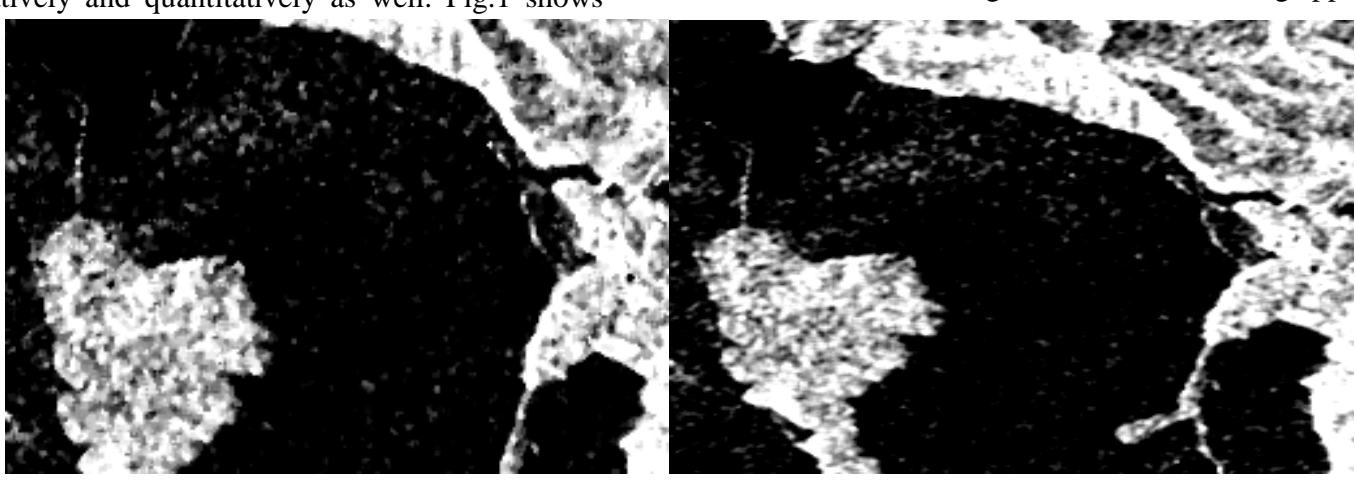

Median

Lee
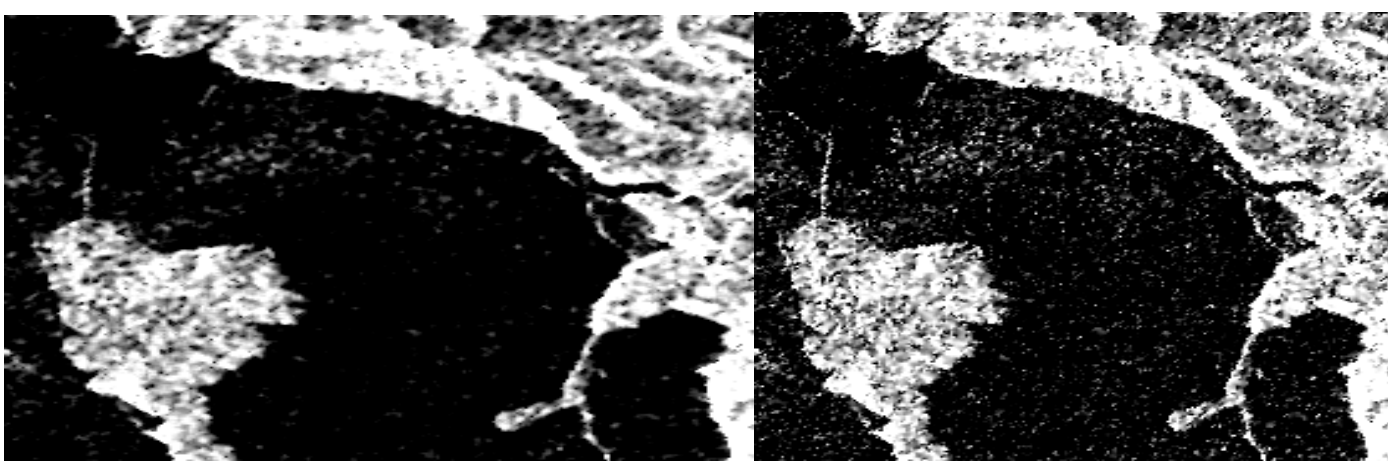

Lee-Sigma

Frost
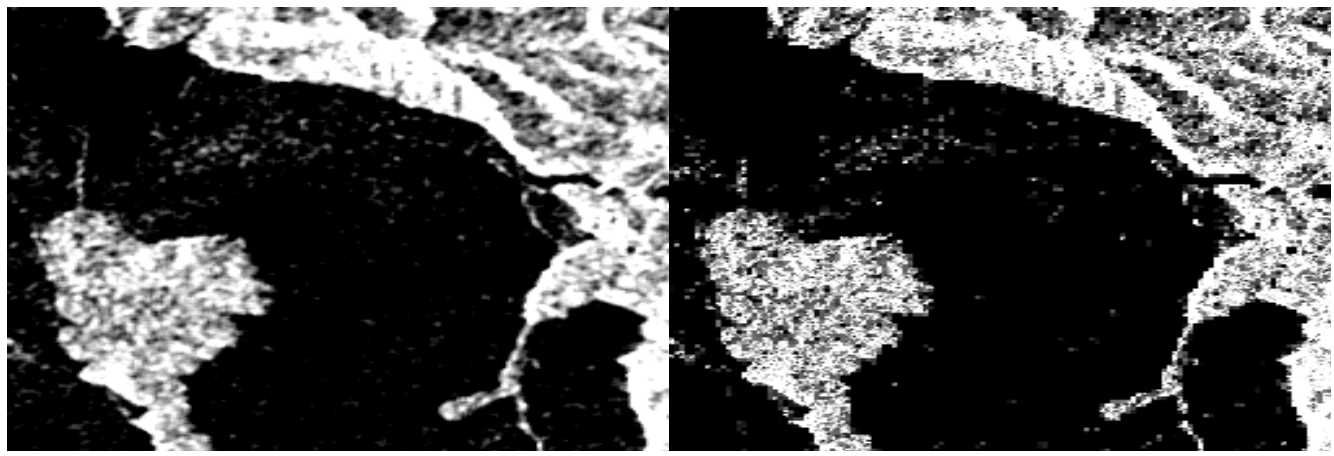

Gamma-MAP

Wiener

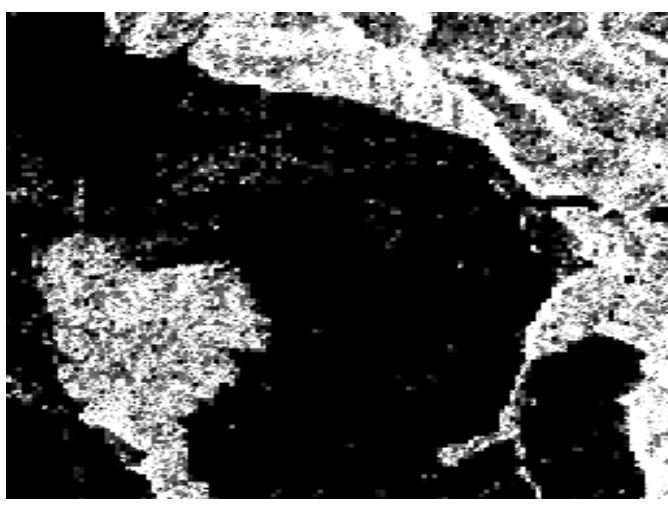

Wavelet threshold

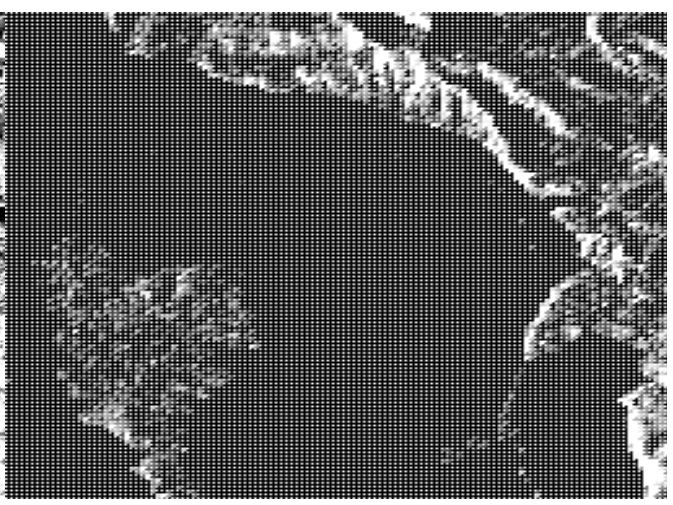

ISPW

Fig1: Qualitative result of various filters 
Table1. Metrics wise Performance comparison

\begin{tabular}{|l|l|l|l|l|l|}
\hline Filters & RR & ENL & SNR & PSNR & EPA \\
\hline Original & 1.4689 & 1.5920 & --- & --- & --- \\
\hline Media & 1.2537 & 2.2796 & 14.2822 & 22.7056 & 0.4241 \\
\hline & & & & & \\
\hline Lee & 1.2384 & 2.3786 & 15.0376 & 23.4610 & 0.4035 \\
\hline Lee-Sigma & 1.2840 & 2.2393 & 15.3985 & 23.8219 & 0.4567 \\
\hline Frost & 1.3656 & 1.9578 & 21.0488 & 29.4722 & 0.7286 \\
\hline Gamma-MAP & 1.2322 & 2.4039 & 14.7218 & 23.1452 & 0.3864 \\
\hline Wavelet threshold & 2.5968 & 0.5030 & 2.3372 & 10.7606 & 0.2755 \\
\hline Wiener & 1.2774 & 2.2910 & 15.2190 & 23.6425 & 0.4438 \\
\hline ISPW & 1.2086 & 2.0171 & 15.7170 & 24.1404 & 0.6103 \\
\hline
\end{tabular}

Table 2. Detailed summary of various methods of denoising

\begin{tabular}{|c|c|c|c|}
\hline $\begin{array}{c}\text { ALGORITH } \\
M \\
\text { IMPLEMEN } \\
\text { TED YEAR } \\
\end{array}$ & $\begin{array}{c}\text { METHOD } \\
\text { USED IN THE ALGORITHM }\end{array}$ & $\begin{array}{c}\text { PERFORMANCE } \\
\text { METRIC } \\
\text { USED IN THE } \\
\text { ALGORITHM } \\
\end{array}$ & $\begin{array}{l}\text { IMAGE/ MODEL } \\
\text { USED IN THE } \\
\text { ALGORITHM }\end{array}$ \\
\hline 1985 & Adaptive noise smoothing & SNR & $\begin{array}{l}\text { NMNV } \\
\text { (darwin1985) }\end{array}$ \\
\hline 1994 & Thresholding method & $\begin{array}{l}\mathrm{s} / \mathrm{m}, \mathrm{t} / \mathrm{c}, \text { target to clutter std } \\
\text { deviation to mean ratio,log } \\
\text { std deviartion deflection }\end{array}$ & $\begin{array}{l}\text { Farm area } \\
(\text { Guo, } 1994)\end{array}$ \\
\hline 1997 & Tree structured filter banks & Noise variance $\sigma$ & Gray image \\
\hline 2001 & Wavelet based adaptive shrinkage & PSNR, shrinkage fn $\gamma$ & Horse track \\
\hline 2004 & Adaptive Median filtering & $\begin{array}{l}\text { SSI,SISA,EEI,FPI,IDPC } \\
\text { (fang qiu,2004) }\end{array}$ & $\begin{array}{l}\text { Radar sat,Jers-1 } \\
\text { dataset }\end{array}$ \\
\hline 2005 & Wavelet based & $\begin{array}{l}\text { MSE,S/MSE, correlation } \\
\text { measure } \beta\end{array}$ & $\begin{array}{l}\text { Autocorrelation } \\
\text { model, Lena and } \\
\text { Barbara }\end{array}$ \\
\hline 2006 & $\begin{array}{l}\text { Bivariate Cauchy distribution } \\
\text { with local variance in CWT }\end{array}$ & PSNR,MMSE & $\begin{array}{l}\text { MR, Lena, Barbara, } \\
\text { boat }\end{array}$ \\
\hline 2007 & Spatially adapted wavelet based & ENL,SSIM,PSNR & $\begin{array}{l}\text { Air SAR image cook } \\
\text { down, collier, } \\
\text { ajkwa }\end{array}$ \\
\hline 2008 & Bivariate shrinkage & R-MSE,SNR,PSNR,ENL & River scene \\
\hline 2009 & Hyper analytic wavelet transform & PSNR & Lena \\
\hline 2009 & Bayesian & MSE,S/MSE, Hypothesis & $\begin{array}{l}\text { 2D } \\
\text { Garch,Aerial1Gamma, } \\
\text { River }\end{array}$ \\
\hline 2010 & $\begin{array}{l}\text { Bivariate Cauchy PDF with MAP } \\
\text { estimation }\end{array}$ & ENL,PSNR,FOM & $\begin{array}{l}\text { Horse track, Road } \\
\text { junction, pip line, } \\
\text { Vegetation, circle }\end{array}$ \\
\hline 2010 & $\begin{array}{l}\text { Institu single pointed wavelet } \\
\text { based }\end{array}$ & $\begin{array}{l}\text { ENL,SNR,PSNR,EPA } \\
\text { RR,(Huangu,2010) }\end{array}$ & $\begin{array}{l}\text { Island area in } \\
\text { china(spot5, Radarsat2) }\end{array}$ \\
\hline 2011 & $\begin{array}{l}\text { Multivariate Cauchy PDF with } \\
\text { MAP estimation }\end{array}$ & ENL,PSNR,MSSIM & $\begin{array}{l}\text { Horse track, } \\
\text { Switzerland, Thal, } \\
\text { circle }\end{array}$ \\
\hline
\end{tabular}


Table 3. Limitations of the various SAR image denoising methods

\begin{tabular}{l}
\hline Denoising methods \\
\hline $\begin{array}{l}\text { Multilook integration technique (Jennifer } \\
\text { Ranjani and Thiruvengadam,2010) }\end{array}$ \\
$\begin{array}{l}\text { Adaptive image restoration technique } \\
\text { (Jennifer Ranjani and Thiruvengadam,2010) }\end{array}$
\end{tabular}

(Jennifer Ranjani and Thiruvengadam,2010)

Spatial Filtering (Huan Gu et al,2008)

Temporal Filtering (Aleksandra,pizurica and wilfried Philips,2001)

Anisotropic diffusion filtering (Venkata Rukmini, 2008)

Bilateral and Trilateral Filtering

(Tomasi and Manduchi, 1998,Choudhury and Tumblin, 2003)

Wavelet transform

.(Marc Simard,1998)

Non Local Mean

(Florence Tupin,2011)

Statistical Approach

(Florence Tupin,2011)

DTCWT (Jennifer Ranjani

and Thiruvengadam,2010)

Curvelet transform

(Krishna Mohan)

\section{Limitations / Disadvantages}

Increase in computational complexity if number of looks increases and thus degrading image resolution.

Blurring and degradation in image resolution if size of window increases.

Blurring edges by averaging pixels and also suitable only for Gaussian noise

Selecting frequency is important and improper selection results aliasing problem

Could not able to retrieve small feature details and yields staircase like pattern in the output image

Large scale structures are only preserved whereas small structures are considered as noise and are removed.

Artifacts are introduced if the selection of threshold of the wavelet is not proper

Computational burden due to its complexity of calculating the weight of the pixel / voxel.

Dephased returned back energy from the target may have phase error

Introduces blurring when decomposition level increases

Poor performance in smooth areas, produce curve let-like artifacts and not efficient if noise is more 
Table 4.Merits of the various SAR image denoising methods

\begin{tabular}{ll}
\hline \multicolumn{1}{c}{ Denoising methods } & \multicolumn{1}{c}{ Merits / Advantages } \\
\hline Multilook integration technique & L looks average used to reduce speckle \\
Adaptive image restoration technique & $\begin{array}{l}\text { Residual speckle is processed using post image formation filters like Lee, MAP etc } \\
\text { adaptive to the adaptive to the local texture information,smooths speckle in } \\
\text { homogenous areas and processing texture and high frequency information in } \\
\text { heterogeneous areas. }\end{array}$ \\
Spatial Filtering & Good in Speckle reduction for small windows size. \\
Temporal Filtering & Applicable for pixel wise process and detail preservation in still image and economical, \\
& less complexity due to time domain
\end{tabular}

Anisotropic diffusion filtering

Non-iterative edge preservation, chosen conduction coefficients smoothes edges and boundaries and better for piecewise constant solutions

better for piecewise constant solutions

Bilateral and Trilateral Filtering

Stronger noise reduction \&better outlier rejection in high gradient rejoin

Wavelet transform

Supports MRA, provides both time \& frequency information.

Non Local Mean

Statistical Approach

DTCWT

Curvelet transform

Contourlet transform (Gangyi Jiang,2006)
Set of similar patches used to suppress the noise and the similarity is based on Euclidean distance between two the patches.

Mellin framework which deals with positive random variables and their multiplication takes into account the variability of the scene to suppress signal-dependent speckle variability.

Improved directional selectivity, shift invariance and perfect reconstruction

Improved and extended wavelet technique which helps to represent multiscale image and sparse representation of objects with edges, wave propagators and in severely illposed problems effectively.

Filter that deals effectively with piecewise smooth images with smooth contours, edges, boundaries and can capture the intrinsic geometrical structure of an image.

\section{CONCLUSION}

In this paper we have mainly focused on various speckle reduction techniques and their merits and demerits. A detailed comparative study of standard spatial domain speckle filters and wavelet domain speckle filters with respect to several metrics have been discussed. The recent developments using advanced image processing concepts such as patch similarity, statistical modeling, Graph cut methods;(Florence Tupin,2011) total variation minimization method and compressed sensing methods have been presented. It has been planned to solve the limitations of increased computational complexity when decomposition levels increased and it has also been planned to improve resolution and speed of operation in a better way. There is always a tradeoff between increase in levels of decomposition and effect of blurring. By increasing number of decomposition levels, one can get more information but this is accompanied by blurring. To get more detailed reconstruction image with edge preservation, less algorithmic complexity and also to improve certain metrics like the ENL, PSNR, Visual quality and best indicator of MSSIM, is a real challenge for the image processing community.

The new Fusion method proposes the use of directionlet transform along with DTCWT. (Jennifier Ranjani and Thiruvengadam,2011) and Non Local means (NLmeans)(Parrilli et al,2010), as the directionlets trace the discontinuity efficiently with fewer significant coefficients for many directions (Biau hou ,2005). The advantages of the proposed method could be the efficient and precise representation of highly anisotropic objects like contours and edges. The other advantage could be the less computational complexity and detailed preservation due to less number of 
processing coefficients. It has been planned to attain improved results over maximum number of metrics without loss of crucial information present in the image without affecting itsoriginality. Compressed sensing and Empirical mode decomposition Algorithms are the imaging trend in speckle noise denoising and have a future scope for the signal processing community.

\section{REFERENCES}

[1] Alin Achim,Panagiotis Tsakalides and Anastasios Bezerrianos 2003, SAR Image denoising Via Bayesian wavelet shrinkage based on Heavy tailed modeling,IEEE trans on GARS,pp1-32.

[2] Albert Cohen and Jelena Kovacevic, 1996, Wavelets: The Mathematical Background, proc of IEEE, 84, pp.514-522.

[3] Aleksandra,pizurica and wilfried Philips,2001,Despeckling SAR images using wavelets and a new class of adaptive shrinkage estimators,J. IEEE,pp.233-236.

[4] Aleksandra,pizurica and wilfried Philips,2001,Noise Reduction in Video Sequences Using Wavelet-Domain and Temporal Filtering,Ghent University, Dept. Telecommunications and Information Processing,SintPietersnieuwstraat 41, B-9000 Ghent, Belgium,pp1-12.

[5] AntoniBuades and Jean-Michel Morel,A non-local algorithm for image denoising.

[6] AntoninChambolle,1998,Nonlinear wavelet image processing:Variational problems,compression, and noise removal through wavelet shrinkage,J.IEEE trans. on image processing,7,pp.319-335.

[7] Biau Hou,2005,SAR Image despeckling based on Improved Directionlet domain Guassion Mixture Model

[8] Bhuiyan,2007, Spatially Adaptive Wavelet-Based Method using the cauchy prior for denoising the SAR images,J.IEEE trans. on circuits and Systems for video Technology, 17,pp.500-507.

[9] Choudhury and Tumblin, 2003, The trilateral filter for high contrast images and meshes, Proceedings of the 14th Eurographics workshop on Rendering, Eurographics Association Aire-la-Ville, Switzerland, Switzerland,pp. 186-196.

[10] Darwin,1985,Adaptive noise smoothing filter for images with signal-Dependent noise,IEEE trans. pattern Analysis and machine intelligence,7,pp.2165-177.

[11] Duan Xinyu and Gao Guowei,2008,A Novel wavelet based Denoising method of SAR image using interscale dependency,,Intl. conf. on computer science and information Technology,pp.889-892.

[12] Ercan kuruglu and Josiane Zerubia Modeling, 2004,SAR images with a generalization of the Rayleigh distribution, IEEE trans. on image processing,13,pp.527-533.

[13] FabrizioArgenti,2002,Speckle removal from SARimages in the Undecimated wavelet domain, IEEE trans. geosciences and remote sensing,40,pp.2363-2374.

[14] Fang Qiu and Judith Berglund,2004,Speckle noise Reduction in SAR Imagery using a Local adaptive
Median filter,J.Geo. science and remote sensing,41,pp.244-249.

[15] Florence Tupin, 2011,How advanced image processing helps for SAR image restoration andanalysis,J.IEEE Geo. and Remote sensing,pp.10-

[16] Gagnon and Jouan,1997,Speckle Filtering of SAR images-A comparative study between complex-waveletbased and standard Filters, SPIE proc,conf. San Diego,Canada.

[17] Gangyi Jiang et al,2006,A New denoising method with contourlet transform,springer pp626-630.

[18] George Tzagkarakis and Panagiotics Tsakalides,2009,Bayesian compressed sensing of a highly impulsive signal in Heavy- tailed noise using a multivariate Cauchy prior, $17^{\text {th }}$ European signal proc. Conf.,pp.2293-2297.

[19] Guo,1994,Wavelet based speckle reduction with application to SAR based ATD R,proc.ICIP,pp1-5.

[20] Guozhong Chen,2006,An improved wavelet based method for SAR images denoising usingFusion techniques, IEEE conf.Radar

[21] Hossein Rabban, 2006,Image Denoising Employing a bivariate Cauchy distribution with local variance in complex wavelet domain, J.IEEE,pp.203-208.

[22] Hua xie,2002,Statistical Properties of Logarithmically transformed speckle,J.IEEE Trans. On Geoscience and Remote Sensing ,40,pp.721-727.

[23] Hua Xie,2002,SAR Speckle reduction using wavelet denoising and Markov random field modeling, IEEE Trans.on Geoscience and remote sensing 140,pp.21962212

[24] Huan Gu,Guo Zhang,and Jun Yan,2008,An INSTITU Single pointed wavelet based method for noise reduction in SAR images, Wuhan univ wuhan,china, The int.Archives photogrammetry,Remote sens. and spatial inf. sciences,pp.337-342

[25] Ioana Firoiu and Corina Nafonita,2009,Image Denoising using a New Implementation of theHyper analytic wavelet Transform,J.IEEE Trans. on instru. And measurement,58,pp.2410-2416.

[26] Jennifer Ranjani and Thiruvengadam,2010,Dual Tree complex wavelet Transform Based SAR Despeckling using Interscale Dependence,J.IEEE Trans. Geoscience and remote Sensing 48,pp.2723-2731.

[27] Jennifier Ranjani and Thiruvengadam, 2011,Generalized SAR Despeckling based on DTCWTexploiting Interscale and Intrascale dependences, J.IEEE trans.on Geoscience and Remote sensing letters, 8,pp.551-555

[28] Johannes Sveinsson and Jon atli Benedikiktson,1996,Tree structured filter banks for Speckle reduction of SAR images, J.IEEE,pp.501504.

[29] Johannes Sveinsson and Jon Atli Benediktsson,1996,Speckle reduction and Enhancement of SAR Images in the Wavelet Domain,J.IEEE, pp.6366. 
[30] Krishna Mohan,Improved Denoising of SAR speckle Images using Curvelets over DT-CWT and traditional filters.

[31] Levent sendur and Ivan W.Selenick,2002,Multivariate shrinkage functions for wavelet based denoising,J.IEEE,pp.953-957.

[32] Majumdar and ward,2009, Sparsity Promoting Speckle denoising"Academia.edu,Intl conf image process.

[33] Marc Simard,1998,Analysis of speckle noise contribution on wavelet decomposition of SAR images,J.IEEE trans. geosciences and remote sensing,36,pp.1953-1962.

[34] Marc Simard,Extraction of information and Speckle noise reduction in SAR Images using the wavelet transform,NASA-Jet propulsion lab,USA.

[35] Maryam Amitrmazlaghan,2009,Speckle Suppression in SAR images using 2-D GARCH model, IEEE trans. on Image Processing ,18,pp.250-259.

[36] Nelson Mascarenhas,1996,An overview of Speckle noise Filtering in SAR image, Proc latino-American seminar on Radar remote sensing, pp.71-79.

[37] Nick kingsbury, 2001,Complex wavelets for Shift Invariant Analysis and Filtering of signals, Applied and computational Harmonic analysis, 10,pp.234-253.

[38] Odegard, 1995, Wavelet based SAR speckle reduction and image compression,proc.of SPIE,pp.1-13.

[39] Pengcheng Han and Jumping Du,2012,spatial image Feature extraction based on Bayesian Non Local Means Filter and Improved Contourlet ransform,Journal of Applied athematics,doi:10.1155/2012/467412,pp116.
[40] Parrilli et al ,2010,A Non Local Approach for SAR Image denoising,DIBET, university Federico II of Naples,Italy,IEEE IGARSS, pp1-4

[41] Radhika,2011, Despeckling of oil spill SAR images using Fusion techniques,Intl J.Computer Applications, 21,pp.0975-8887.

[42] Shuai xing, 2008,Speckle denoising based bivariate shrinkage functions and Dual tree complex wavelet transform", the international archives photogrametry,remote sensing and spatial information sciences,37,pp.157-161.

[43] Syed Musharaf,2007,Wavelet Based Despeckling of synthetic aperture radar images using Adaptive and Mean filters, World Academy of science and tech,31,pp.39-42.

[44] Tomasi and Manduchi, 1998,Bilateral filtering for gray and color images,Sixth International Conference on Computer Vision,pp. 839-846.

[45] Venkata Rukmini,2008,Thesis on Filter selection for Speckle noise Reduction,Thapar university,Patiala,Punjab.

[46] Wangwen Xing Fu-cheng,2005,Speckle reduction of SAR image based on modeling autocorrelation function of wavelet coefficients,IEEE international symposium on microwave, antenna propagation and EMC Tech for wireless Proceedings,IEEE,pp.383-386.

[47] Zengguo sun and Chongzhao Han, 2006, MAP Filtering for SAR images based on Heavy tailed Ray leigh modeling of speckle, IEEE, pp.323-328.

[48] Zhou Wang and Alan Courad Bovik, 2004, Image Quality Assessment: From Error Visibility to Structural similarity, J.IEEE Trans. on Image Processing, 13, pp.600-612. 